\title{
Faire connaître et aimer la littérature en classe
}

Un exemple en France

Appreciating and enjoying literature in the classroom. An example in France

Dar a conocer y amar la literatura en clase. Un ejemplo en Francia

\section{Anne Maurel}

\section{OpenEdition}

\section{Journals}

Édition électronique

URL : https://journals.openedition.org/ries/2109

DOI : 10.4000/ries.2109

ISSN : 2261-4265

\section{Éditeur}

France Education international

\section{Édition imprimée}

Date de publication : 1 septembre 2011

Pagination : 147-153

ISBN : 978-2-85420-592-3

ISSN : $1254-4590$

Référence électronique

Anne Maurel, « Faire connaître et aimer la littérature en classe », Revue internationale d'éducation de Sèvres [En ligne], 57 | septembre 2011, mis en ligne le 01 septembre 2014, consulté le 06 juillet 2021. URL : http://journals.openedition.org/ries/2109; DOI : https://doi.org/10.4000/ries.2109 


\section{Faire connaître et aimer la littérature en classe}

\section{Un exemple en France}

\section{Anne Maurel}

On peut préférer - et c'est mon cas - se dire professeur de lettres plutôt qu'enseignant de français. On écarte ainsi une visée trop strictement utilitariste et instrumentale. On s'attache à nommer la littérature et on se donne pour but de la faire connaître et aimer. Reste un obstacle à la fois extérieur à la classe et interne aux représentations. L'ennui, ici, n'est pas seulement celui des temps et des lieux quadrillés. L'ennui précède la classe et on pressent, corrélativement, que le «plaisir » de la littérature excède l'agrément, souhaité et souhaitable, des lieux. Mais c'est bien dans la classe que quelque chose peut avoir lieu.

Un texte classique, c'est-à-dire à la lettre enseigné en classe, est comme « embaumé » et «tenu en respect. » Il s'est créé entre lui et des élèves une distance qu'on suppose difficile à franchir, tant en raison de l'éloignement du temps et de l'étrangeté relative de sa langue que de la sacralisation, par une communauté nationale, de sa beauté. Ce que traduit la majuscule dont on orne parfois le mot Littérature. On y entre comme dans un temple. Et s'il arrive que des élèves manifestent un certain plaisir dès l'entrée, à la simple mention du nom de l'écrivain c'est bien souvent pour signer leur appartenance au groupe des lettrés et autres gens bien nés. Un goût déclaré pour des valeurs reconnues comme des valeurs sûres de notre culture (le roman de Proust ou les tragédies de Racine) fonctionne comme un signe de reconnaissance. L'idée s'est ainsi répandue que la bourgeoisie aurait plus aisément, voire plus naturellement, accès à la littérature que des enfants issus de milieux sociaux moins favorisés. Or ma pratique de l'enseignement dans des lieux aussi opposés que le sont des classes de collèges et de lycées polyvalents du département de la Seine-SaintDenis, dans lesquelles j'ai enseigné de 1975 à 1990, et des classes préparatoires littéraires du Lycée Henri IV, où j'exerce depuis une vingtaine d'années, a assez largement infirmé ce préjugé. Car partout et pour tous, une œuvre littéraire qu'on dit " classique » est d'abord lettre morte. Elle vaut pour sa valeur d'échange avant que de valoir pour elle-même, et ce quels que soient le degré d'enseignement et l'origine sociale des élèves. La voix qui parle en elle a été étouffée et recouverte par un discours académique transmis de génération en génération. À chaque grand nom, on a attaché une série de chromos et d'images d'Épinal.

La question qui se pose à l'enseignant est dès lors partout la même. Comment faire pour que le théâtre de Corneille ne se résume pas à jamais en 
cette unique formule de " dilemme cornélien », de conflit de la volonté avec la passion, qui lui donne la raideur héroïque et la couleur surannée des anciens temps ? Comment faire entendre la violence des sentiments qui se cache sous l'extrême politesse des vers de Racine? Quels moyens utiliser et par quelles étapes doit-on passer pour ouvrir des trouées dans l'admiration protocolaire que nous portons aux chefs d'œuvre de notre panthéon national ? Comment rendre possible, dans la classe, la rencontre d'un lecteur singulier avec une œuvre singulière?

D'expérience, la question est la même, dans des situations que l'on sait opposées à l'extrême : elle se pose différemment, mais c'est bien la même, que l'on accueille une classe de quatrième, une première technique, ou que l'on travaille le programme d'un concours dans une classe de khâgne. On sait maintenant ce qu'il faut de luttes, d'endurance et de résistance, de souffrance parfois et d'héroïsme, particulièrement dans les collèges, pour surmonter la difficulté et conduire des élèves à devenir des lecteurs. On recense les obstacles et on recherche avec raison des conditions plus favorables. Mais favorables à quoi ? À l'événement de la rencontre avec un texte. Le défi est le même à l'autre extrémité de l'institution scolaire française, car on n'a peut-être pas encore pris la mesure de ce que la motivation au travail des hypokhâgneux et des khâgneux réussit pourtant mal à masquer : dès avant le début de la classe, l'angoisse d'échouer 148 les a précipités dans des formalismes fallacieusement "payants » et à coup sûr " mortels ", dont il est extrêmement difficile de les détacher. Ainsi, pour ceux qui sont censés être une élite, l'académisme achève l'enterrement de la lettre morte. Tant et si bien qu'ils sont désormais nombreux à dire, lorsqu'on les interroge, qu'il leur faut, une fois le concours passé et la réussite obtenue, faire des efforts considérables pour penser et écrire en dehors des cadres étroits d'une rhétorique scolaire, pour animer leur plume et revivifier les livres lus.

La classe est donc comme cernée par le système. L'étude de la littérature à l'école tente bien de jeter un pont entre la littérature et la vie. Mais force est de constater que l'organisation, tout au long de la scolarité, des cours de lettres a quelque chose du catéchisme. Notre histoire littéraire est présentée comme une légende, au sens premier de ce terme : un livre contenant les actes des saints pour toute l'année, ainsi appelé parce qu'à certains jours on désignait la portion qui devait être lue - legendum. On doit lire les fables de La Fontaine à l'école primaire et jusqu'en sixième ; le théâtre du grand siècle, Corneille, Molière et Racine, en quatrième ; les grands poètes du XIX siècle en première ; Montaigne et Pascal dans les classes de terminale. Chaque âge a ainsi accès, sans accident ni surprise, à une tranche du patrimoine national. La mémoire scolaire est un tissu serré de citations, apprises par cœur ou qui se sont spontanément gravées dans nos esprits, que nous associons à une saison de la vie et aux émotions censées leur correspondre. Les premiers émois amoureux trouvent dans les Nuits de Musset une orchestration grandiose. "Le silence de ces espaces infinis 
m'effraie » sonne à l'unisson des inquiétudes métaphysiques de l'adolescence, et ainsi de suite. Il est alors difficile de faire de la littérature ainsi enseignée autre chose qu'un magasin aux accessoires, une grande réserve de clichés dont on fera usage en toutes circonstances. La formule originale n'a rien perdu de sa vigueur - c'est pourquoi on la retient - mais on a cessé, à force de la répéter, de l'entendre. Son sens s'est décoloré.

L'ennui que l'on peut éprouver dans une classe de littérature tient ainsi pour beaucoup à des causes extérieures à son objet : ce sont des raisons morales et sociales. Car faire de la littérature un moyen d'édification ou, de façon plus étroite, un viatique pour briller dans certaines sociétés choisies, c'est immanquablement se condamner à rester enfermé dans un discours convenu et conventionnel sourd à ce qui pourtant caractérise un texte de littérature: son irréductible singularité. Aucune œuvre de littérature ne saurait être réduite à un résumé, à une traduction, pas plus d'ailleurs qu'au repérage de ses structures ou à la mise au jour de ses codes. Sa valeur intrinsèque tient à son aptitude à toucher son lecteur, à le déranger, à l'affecter souvent en quelque région le plus souvent secrète, intime, de lui-même, voire à le transformer. Et c'est à découvrir et à faire découvrir, ou plutôt à expérimenter et à faire expérimenter par ses élèves cet effet propre au texte de littérature que le professeur de lettres peut désirer travailler.

Comment traverse-t-on un tel ennui ? Parfois par ruse, et parfois en le forçant. On n'est jamais assuré de le faire. Plus que des étapes (et quoi que l'on en prévoie), il se produit des brèches, soulageant l'inquiétude ou délivrant un temps l'angoisse du professeur. Ce sont des trouées, des éveils, des amorces. Un processus commence, à la fois très personnel, pour l'élève, mais accueilli dans le lieu de la classe en commun, où a lieu une saisie : l'attention de tous se produit, comme un événement et non plus comme une obéissance autoritairement requise.

Ce je ne sais quoi est lié à ce qu'éprouve celui qui enseigne. S’il s'ennuie, " ils s'ennuieront ». Quant au "plaisir » ou à l'intérêt qu'il recherche, on peut supposer qu'il est aussi perçu par les élèves, mais le décisif est leur propre saisie, leur propre expérience directe, sensible, faite à la fois en commun et en solitude : c'est en marchant que l'on découvre les surprises d'une route, ses tournants, ses lointains et ses perspectives sans cesse renouvelées, et ils sont de plusieurs ordres. Ma passion pour l'exercice du com-mentaire (avoir un texte en esprit - mens - avec soi, l'avoir comme compagnie) est sans doute décisive, mais elle ne devient "communicative » que dans le fait que les élèves en font la découverte et l'expérience. Celle-ci se fait de plusieurs manières, en plusieurs strates d'attention ou de résonance.

L'expérience de découverte attentive du texte dans sa présence et sa tournure est ce que je pratique et soutiens chez mes étudiants, contre le pédantisme. J'ai souvent fait ce rêve que mes élèves apprennent par l'activité du commentaire 
à se frayer de nouveaux chemins dans une œuvre classique. Bien sûr, ou sans doute, faut-il en savoir quelque chose. Mais une fois un texte survolé, compris selon les mêmes lois que le terrain qui l'entoure (situé dans son époque, avec sa morale, son système de valeurs, sa langue et ses formes), je cherche à faire qu'on le lise de nouveau, à nouveau, en éprouvant et en exprimant des étonnements, en arrêtant son attention sur ce qu'il y a en lui d'inouï. Chaque nouveau lecteur est un premier lecteur. Chaque lecture est une attention neuve. Où l'on peut à la fois soutenir la fraîcheur du lecteur novice, et exercer son attention pour saisir ce qu'un texte offre et exige à la fois, dans sa concision même. Dans les Pensées, par exemple, j'attire leur attention sur tel très court fragment « Son bec que le perroquet essuie, même s'il est sec. » Sa brièveté et son allure volontairement énigmatique, la façon dont il contraste avec les développements longs et articulés sur les deux infinis, (l'infiniment petit et l'infiniment grand de Pascal transformés eux aussi en, clichés !), sont bien propres à arrêter l'esprit et à l'engager dans une activité d'observation et d'interprétation. C'est le moment de se poser des questions, de découvrir de nouvelles zones d'intérêt et de nouveaux critères de jugement de l'œuvre. Mais avant même cela, il s'agit de se laisser exposer au texte même, au tissage et à l'intrication des mots qui sont là, et eux seuls. Ici, par exemple, la forme ramassée produit un impact sonore. La pensée, d'abord, se refuse. Alors on examine ce qui est là. La syntaxe est une syntaxe de l'œil : elle oblige le lecteur à participer à ce qui est dit, à se transporter sur une autre scène, celle que le texte instaure, pour y voir ce qu'il n'a pas encore vu parce qu'il néglige trop souvent dans la vie ordinaire de prêter son attention à des détails qu'il juge lui-même infimes et insignifiants, jusqu'à ce que leur profondeur lui soit révélée par la lecture d'un texte de littérature. Des mots quelconques acquièrent une nouvelle puissance de signifier : deux pauvres mots d'une seule syllabe, bec, sec ouvrent et ferment la phrase en se répondant en écho dans une rime intérieure et voilà que nous éprouvons, que nous entendons presque, l'abîme entre l'homme qui peut penser, et l'animal machine qui essuie sans raison son bec sec, voilà que nous comprenons même la vanité de l'homme qui peut aussi devenir mécanique, bec et bête, et faire le perroquet.

Il est à noter que l'on est loin, bien loin, ici, du supposé « beau langage » trop souvent censé signaler la Littérature et participer du plaisir du texte pour quelques uns, mais pour quelques uns seulement, en tenant écartés de ce plaisir des lecteurs moins habitués à tant de raffinement langagier. Au contraire, le plaisir du com-mentaire qui nous fait accompagner un texte par la pensée et entrer dans son jeu avec la syntaxe, avec le volume et les sons des mots, aussi simples qu'ils soient, est un plaisir sensible et partageable par tous. C'est le plaisir que l'on éprouve à voir le monde d'un regard neuf, à voir autre chose, autrement ; le plaisir que l'on a à sortir des sentiers battus. Plaisir de l'élève et tout ensemble du professeur. Le plaisir de l'élève est le plaisir d'aller vers la lecture : de mettre ses pieds dans les traces de l'écrivain et de collaborer avec lui. C'est un plaisir actif qui se prolonge au-delà de la lecture d'un texte : 
apprendre à entrer à chaque fois dans un monde autre, celui du texte. L'esprit acquiert ainsi plus d'agilité qu'il n'en a dans la vie courante. Il arpente et explore de nouveaux territoires jusque là inconnus. Le plaisir du professeur tient à ce qu'il parvient ainsi à ce que le cours de littérature, qui a malheureusement abandonné l'exercice du pastiche depuis la fin du XIX ${ }^{\mathrm{e}}$ siècle, renoue, par le commentaire, avec les vertus de la copie. Pasticher peut être très amusant et apprend beaucoup du texte que l'on imite, avec plus ou moins de distance joueuse (joyeuse), et copier - les peintres le savent - apprend à regarder, permet de voir ce qu'on n'avait pas remarqué. En s'essayant à écrire sans glose, au plus près de ce qu'on lit, on éprouve en devenant attentif. On ne résume plus une œuvre, mais on saisit l'énergie d'une écriture.

Plaisir de l'intelligence, de l'oreille, et de l'œil, ce n'est pas un jeu de " pur esprit ", superficiel, formel ou désincarné. Il faut prendre la mesure de ce qu'une telle expérience est une sorte d'exercice spirituel, en un sens à la fois tout à fait laïque, et sensible : un lieu où l'esprit se saisit de la vie même, et où un être, quel qu'il soit, élève ou professeur, est saisi par une forme ou une formulation qui soudain le concerne, parce qu'elle lui parle sans complaisance de ce que c'est qu'exister.

Il peut ainsi arriver que ce plaisir pris au texte soit occasion pour certains d'entendre soudain dans une œuvre des échos aux questions qui leur sont propres, voire d'y trouver des issues possibles aux impasses où la vie les a parfois momentanément placés. Alors, l'étude du lien entre la forme et le fond donne lieu à un lien entre le texte et la vie. La lecture et le commentaire de textes extraits du Discours sur les sciences et les arts dans une classe de première technique du lycée de Villepinte, en Seine Saint Denis, où venait d'arriver un élève qui avait passé trois années en prison, a ainsi donné lieu à un développement sur la pensée paradoxale de Rousseau. Ce très jeune homme s'est immédiatement emparé d'une notion pour lui nouvelle, le paradoxe, m'en demandant aussitôt une définition la plus précise possible, et me demandant avec, à ce qu'il me semblait, une certaine jubilation, de la lui répéter. Appuyé sur cette découverte, il a radicalement transformé le regard du groupe sur lui. Cette même année, ayant pris la tête d'un groupe d'élèves qui exposaient leurs revendications devant les parents et l'administration du lycée, il a, de façon assez inattendue, emporté l'adhésion d'une assemblée qui lui était jusque là hostile et qui, quelques mois auparavant, demandait son éviction de la classe. À cette expérience mémorable de la rencontre d'un élève avec un texte de littérature venu pour lui à point nommé, je pourrais ajouter une anecdote plus légère, mais elle aussi significative des résonances qu'une œuvre peut avoir dans une classe. La lecture d'une scène de Suréna, où un Prince amoureux et soupçonneux traque le secret amour pour un autre de la femme qu'il convoite, lui dictant ce qu'elle lui dirait si elle l'aimait, a conduit un élève d'une classe d'hypokhâgne à s'interroger, non sans inquiétude, sur la part, dans le discours amoureux, de l'éloquence spontanée, 
inspirée par la vivacité du sentiment, et de la rhétorique apprise, et sur la difficulté à démêler l'une de l'autre.

Enseigner le texte plutôt que l'histoire littéraire, ou, à tout le moins, refuser de faire du texte, du " morceau choisi », l'illustration simple des grandes lignes de l'évolution littéraire; en retrouver par soi le parcours au lieu de le survoler, c'est arracher l'enseignement de la littérature à la monotonie et à l'ennui des propos sans cesse répétés. C'est l'ouvrir au plaisir de la découverte. Mais c'est donc, plus encore, permettre que se produisent des rencontres décisives entre une œuvre de littérature et un lecteur. Quand Kafka proposait qu'on ne lût que « des livres qui mordent et qui piquent », et qu'un livre fût « la hache qui brise la mer gelée en nous ", c'est qu'il avait en tête qu'un livre véritablement et activement lu, c'est-à-dire parcouru comme on parcourt une route à pied, pour ouvrir dans le paysage des perspectives inconnues, peut nous aider à frayer des routes dans l'étendue obscure ou glacée de nos émotions intimes.

Comment se fait pour le lecteur, en classe, cette "jonction » entre le texte et la vie? En classe préparatoire, je n'ai pas le loisir de choisir les textes que je commente, imposés par le programme du concours, au contraire de ce que je faisais dans les collèges et lycées de banlieue. Mais lorsque cela est possible, ou lorsqu'on s'en accorde la liberté, la recherche d'un texte pour une classe est comme le plaisir de préparer un banquet pour ses hôtes. Telle page de Moby Dick, la rencontre dans un même lit d'auberge d'Ismael, le narrateur, avec l'Indien Queequeg; ou bien encore la totalité d'un petit livre de Victor Hugo qui "fait mal aux nerfs » au dire même de son auteur, Le Dernier jour d'un condamné, captent l'attention des élèves sans que j'aie besoin de trop d'efforts. Au point qu'on peut se demander si des œuvres qui se saisissent de la vie, et de sa dureté, ne seraient pas aptes, plus même peut-être que des lectures légères et plaisantes, à produire cette rencontre avec la littérature d'adolescents moins désireux qu'on ne le pense ordinairement de ne lire que des histoires où tout se répare toujours.

Mais alors, découvrir dans un livre comme une bribe du texte de sa propre vie, accéder à un tel exercice de l'esprit n'est pas si abstrait qu'on le croit : c'est une épreuve sensible. Et il y faut une dimension sensible et vivante, ni distance, ni « mise en scène » artificielle. Le professeur qui est d'abord lecteur, se fait liseur pour espérer être passeur, et que l'auditeur puisse devenir lecteur à son tour tient à ce que le liseur s'expose au texte, laisse sa voix nue accueillir et faire entendre - sans la forcer ni la singer - la voix oubliée du texte. Si jamais s'est produite pour mes élèves quelque rencontre, je crois que c'est en fait d'abord dans ma voix, mise elle-même à l'épreuve de la lecture à voix haute, où je me laisse moi-même surprendre par la puissance d'évocation de ce qui résonne. Parfois c'est simplement un silence autour du texte qui est l'écrin. Le silence en classe est une épreuve dont le vide peut être effrayant, sidérant, pour les élèves, voire pour le professeur. Se déployant, il laisse le texte disséminer ses 
charges de pensée. Il accorde le temps de méditer les pensées engendrées. La voix reprend, pensive, ranimant dans son défaut même la voix éteinte du texte.

La voix est un vecteur essentiel pour adresser un texte à des élèves quels qu'ils soient et la lecture à voix haute un moment trop oublié, un étrange plaisirépreuve. Mais elle n'agit pas, me semble-t-il, de la même façon dans tous les lieux. Il m'a semblé que dans mes classes de banlieue, ma voix a souvent contribué à rendre plus proche à mes élèves un texte de Littérature (avec la majuscule d'usage). Tout se passait comme s'ils retrouvaient dans ma voix leur lisant une page, ou plus, d'un texte classique, quelque chose de la voix d'une mère leur contant une histoire. Ce qui m'y fait songer c'est, une fois que je lisais une tirade de Don Juan, la remarque très insolite d'une de mes élèves qui avait levé le doigt pour me dire tout à trac « j'aimerais avoir une mère comme vous ", alors que rien dans le texte lu ne supposait que, l'écoutant, l'on pensât à l'enfance. Dans les classes préparatoires, et en particulier l'année du concours, je me sers de ma voix, quitte parfois à la forcer un peu, pour tenter de vaincre l'ennui et la fatigue produites par des lectures contraintes, proposées en trop grande quantité et menées à marche forcée. En rendant perceptible l'effet sur moi d'un texte et en laissant passer dans ma voix ou dans certains de mes silences quelque chose de mon émotion, je cherche à ranimer en eux une écoute sensible, attentive à d'imperceptibles nuances, qui pour être commentées doivent d'abord avoir été entendues. Des têtes se tournent alors vers la voix ; des regards se détachent, un instant au moins, des écrans des ordinateurs. Des paroles parfois viennent, qui sortent le professeur de sa solitude. Dans les petites classes, j'étais aux aguets de ces réponses d'abord presque imperceptibles. Une fois cette vérité du texte rendue sensible, les élèves trouvent la parole, font entendre une voix, parfois dans de savoureux malentendus, que l'on accueille sans moquerie, en se réjouissant des découvertes de libres jeux du signifiant, pour peu que la sanction de l'examen ait pu être un temps mise à distance. La question du formalisme des exercices peut se poser autrement, être mise au service de ce que l'élève a à dire et cherche à formuler.

Et chaque année rejoue à la rentrée cette aventure non assurée.

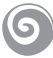

Ainsi ce qui arrache une classe à l'ennui est-il moins l'agrément d'un divertissement, que la rencontre de ce qui, dans un livre, "brise la mer gelée en nous ». S'agit-il d'un plaisir? Dans l'irruption d'un moment d'attention en commun, on a le sentiment - partagé dans la classe - que quelque chose se dénoue, même fugitivement. Cela tient parfois à une inflexion involontaire de la voix attentive. Cela passe par le médiation incarnée du professeur. Ce qu'il vise est-il un plaisir ? Plus profondément, ce qu'il espère, et ce qu'il peut arriver à tous de ressentir, est une joie, celle d'avoir trouvé des mots imprévisibles, parfois pour des tourments. 